\title{
A Novel Model of Charged Leptons
}

\author{
Dianfu Wang, Xiao Liang, Yanqing Guo* \\ School of Science, Dalian Maritime University, Dalian, China \\ Email:wangdfu@dlmu.edu.cn, *yqguo@dlmu.edu.cn
}

How to cite this paper: Wang, D.F., Liang, X. and Guo, Y.Q. (2020) A Novel Model of Charged Leptons. Journal of Modern Physics, 11, 448-454.

https://doi.org/10.4236/jmp.2020.113028

Received: February 21, 2020

Accepted: March 17, 2020

Published: March 20, 2020

Copyright $\odot 2020$ by author(s) and Scientific Research Publishing Inc. This work is licensed under the Creative Commons Attribution International License (CC BY 4.0).

http://creativecommons.org/licenses/by/4.0/

\begin{abstract}
A novel model of charged leptons is presented, which contains two basics hypotheses. The first hypothesis is that the Yukawa coupling between Higgs field and charged leptons is the weak interaction, the Higgs field is a scalar intermediate boson which changes the chirality of charged leptons in the weak interaction. The other hypothesis is that the flavor eigenstates of charged leptons are the superposition states of left-handed and right-handed elementary Weyl spinors before the electroweak symmetry breaking. According to this model, the Yukawa coupling constants between Higgs field and three generations of charged leptons are considered to be a universal constant, and the difference of the masses of different charged leptons is due to the different left-right mixing angles of their flavor eigenstates.
\end{abstract}

\section{Keywords}

Charged Leptons, Weak Eigenstates, Left-Right Mixing Angle

\section{Introduction}

Up to now, with the discovery of the $125 \mathrm{GeV}$ Higgs boson in 2012 at the CERN LHC [1] [2], the Weinberg-Salam (WS) model [3] [4] of the electroweak interaction of particle physics stands triumphant, and almost all relevant experimental results in particle physics are consistent with this model. Although the WS model has achieved impressive success in correlating all observed low-energy data in terms of a very few parameters, it cannot be called perfect. For example, the model is based on too many assumptions and leaves many fundamental questions unanswered. The success of the WS model only involves the gauge sector of the theory, in which only one free parameter, the Weinberg angle $\theta_{W}$, is used to understand numerous neutral-current data. But for the fermionic sector, the fermion mass spectrum ranges from $170 \mathrm{GeV}$ of the top-quark to $0.511 \times 10^{-3}$ $\mathrm{GeV}$ of the electron. We do not know why there exists such a large difference 
among the masses of these fermions, and we have no deep understanding of the origin of these masses. Since the fermion masses are related to the Yukawa couplings, we can only understand these differences and the mass-generation phenomenon by studying the Yukawa couplings in detail [5] [6] [7] [8].

\section{A Charged Lepton Model}

Quarks and leptons constitute the basic building blocks of matter in the standard model. In the WS model, there are three generations of quarks and leptons with identical quantum numbers but different masses. Since quarks are supposed to be confined by virtue of their strong interactions, the meaning of the quark mass is not obvious [9]. Therefore, in the present paper, for simplicity, we will limit ourselves to three generations of leptons. Let's start with discussing the lepton sector. Observationally, we must incorporate a neutral, left-handed neutrino (For simplicity, the neutrino is assumed to be massless, and hence right-handed neutrino is absent.) along with a charged lepton, which can be considered to be the sum of left-handed and right-handed Weyl spinors. The left-handed fermions form an isodoublet, consisting of the neutrino and the charged lepton:

$$
L_{\ell}=\frac{1}{2}\left(1+\gamma_{5}\right)\left(\begin{array}{c}
v_{\ell} \\
\ell
\end{array}\right)=\left(\begin{array}{c}
v_{\ell} \\
\ell
\end{array}\right)_{L},
$$

while the right-handed sector consists of an isosinglet, the right-handed charged lepton:

$$
R_{\ell}=\frac{1}{2}\left(1-\gamma_{5}\right) \ell=\ell_{R},
$$

where $\ell=e, \mu, \tau$ denote the flavor indexes of the charged leptons, and the subscripts $L$ and $R$ refer to the left- and right-handed components, respectively. In the standard WS model, the usual default setting is that the flavor eigenstates of charged leptons are the same as their mass eigenstates (An eigenstate of finite mass is a superposition of left and right-handed states with equal weight [9]), i.e.,

$$
\ell=\ell_{L}+\ell_{R} .
$$

with the chiral fermions given in Equation (1) and Equation (2), the most general $S U(2)_{L} \times U(1)_{Y}$ gauge-invariant lepton part of the Lagrangian density of WS model can be written as

$$
\begin{aligned}
\mathscr{L}_{\ell}= & -\bar{L}_{\ell} \gamma_{\mu}\left(\partial_{\mu}-i g \frac{1}{2} \tau^{i} A_{\mu}^{i}+i g^{\prime} \frac{1}{2} B_{\mu}\right) L_{\ell} \\
& -\bar{R}_{\ell} \gamma_{\mu}\left(\partial_{\mu}+i g^{\prime} B_{\mu}\right) R_{\ell}-G_{\ell}\left(\bar{L}_{\ell} \phi R_{\ell}+\bar{R}_{\ell} \phi^{\dagger} L_{\ell}\right) .
\end{aligned}
$$

By defining the mass eigenstates of gauge fields

$$
\begin{aligned}
W_{\mu}^{ \pm} & =\frac{1}{\sqrt{2}}\left(A_{\mu}^{1} \mp i A_{\mu}^{2}\right), \\
Z_{\mu} & =\sin \theta_{W} B_{\mu}-\cos \theta_{W} A_{\mu}^{3}, \\
A_{\mu} & =\cos \theta_{W} B_{\mu}+\sin \theta_{W} A_{\mu}^{3},
\end{aligned}
$$


where $\theta_{W}$ is the Weinberg angle with $\tan \theta_{W}=g^{\prime} / g$, the interaction term that leptons coupled to gauge fields and Higgs field in Equation (4) can be written as

$$
\begin{aligned}
& \mathscr{L}_{\ell}^{\prime}=+i \frac{g}{\sqrt{2}}\left[W_{\mu}^{+} \overline{\left(v_{\ell}\right)} \gamma_{L} \ell_{L}+W_{\mu}^{-} \bar{\ell}_{L} \gamma_{\mu}\left(v_{\ell}\right)_{L}\right] \\
& -i \sqrt{g^{2}+g^{\prime 2}} Z_{\mu}\left[\frac{\overline{\left(v_{\ell}\right)_{L}} \gamma_{\mu}\left(v_{\ell}\right)_{L}-\bar{\ell}_{L} \gamma_{\mu} \ell_{L}}{2}+\sin ^{2} \theta_{W} \bar{\ell} \gamma_{\mu} \ell\right] \\
& -i \frac{g g^{\prime}}{\sqrt{g^{2}+g^{\prime 2}}} A_{\mu} \bar{\ell}_{\mu} \ell-G_{\ell}\left(\bar{L}_{\ell} \phi R_{\ell}+\bar{R}_{\ell} \phi^{\dagger} L_{\ell}\right) .
\end{aligned}
$$

The last term on the right side of Equation (6) is the gauge invariant Yukawa coupling between Higgs field and fermions,

$$
\mathscr{L}_{\mathrm{Y}}=-G_{\ell}\left(\bar{L}_{\ell} \phi R_{\ell}+\bar{R}_{\ell} \phi^{\dagger} L_{\ell}\right),
$$

where $G_{\ell}$, an additional parameter, gives the strength of the Yukawa coupling. In the standard WS model, all the charged leptons are massless as long as electroweak symmetry is unbroken, and they can get masses from Yukawa interactions only after electroweak symmetry breaking. A convenient way to implement this is to introduce a doublet Higgs field

$$
\phi=\left(\begin{array}{c}
\phi_{+} \\
\phi_{0}
\end{array}\right),
$$

where the subscripts refer to the electric charge. Replacing $\phi$ by its vacuum expectation value

$$
\langle\phi\rangle=\left(\begin{array}{l}
0 \\
v
\end{array}\right),
$$

the mass term of the charged leptons can be given by the Yukawa coupling Equation (7) as

$$
\mathscr{L}_{m_{\ell}}=-G_{\ell} v \bar{\ell} \ell .
$$

Thus we can identify the masses of the charged leptons

$$
m_{\ell}=G_{\ell} v \text {. }
$$

However, it does not specify the value of the masses since the Yukawa coupling constant $G_{\ell}$ is arbitrary. So far, the standard model of the electroweak interaction has not given more further information on the origin of charged lepton masses. Furthermore, it is important to notice that since the strength of interaction between the Higgs field and any particle is proportional to the mass of that particle, this means that there is a new type of interaction that is different from the strong interaction, the electroweak interaction and even the gravitational interaction, which is very unnatural.

Since the mass of the Higgs boson is similar to that of $\mathrm{W}$ and $\mathrm{Z}$ bosons, so we could find it on the LHC in 2012. To some extent, they are all the same particles, or fields that presumably mediate weak interactions. In this sense, the Higgs boson will be no different from $\mathrm{W}$ and $\mathrm{Z}$ bosons. If these considerations are reasonable, the coupling constants of the four interaction terms in Equation (6) will 
be of the same order of magnitude, which means the Yukawa coupling in Equation (4) can be written as

$$
\mathscr{L}_{\mathrm{Y}}=-g^{\prime \prime}\left(\bar{L}_{\ell} \phi R_{\ell}+\bar{R}_{\ell} \phi^{\dagger} L_{\ell}\right),
$$

where $g^{\prime \prime}$ is the universal coupling constant of the Yukawa coupling. Based on the above discussion, we assume that the Yukawa coupling is the weak interaction, and the Higgs field is a scalar intermediate boson which changes the chirality of particles in the weak interaction. Since in low-energy processes the Higgs-meson and the $\mathrm{W}$-meson carry small momentum, the propagators of them may be taken to be $M_{H}^{-2}$ and $M_{W}^{-2}$. By considering Equation (6) and Equation (12), we can then obtain the following relation between the coupling constant $g^{\prime \prime}$ and the gauge coupling constant $g$ as

$$
\frac{g^{2}}{2 M_{W}^{2}}=\frac{g^{\prime \prime 2}}{M_{H}^{2}},
$$

which gives the universal Yukawa coupling constant

$$
g^{\prime \prime}=\frac{g}{\sqrt{2}} \frac{M_{H}}{M_{W}}
$$

Replacing $\phi$ by its vacuum expectation value $\langle\phi\rangle$, Equation (12) changes to be the mass term of the charged leptons

$$
\mathscr{L}_{m_{\ell}}=-g^{\prime \prime} v \bar{\ell} \ell=-\frac{g v}{\sqrt{2}} \frac{M_{H}}{M_{W}} \bar{\ell} \ell,
$$

The massess of the charged leptons derived from Equation (15) can be easily shown as

$$
m_{\ell}=\frac{g v}{\sqrt{2}} \frac{M_{H}}{M_{W}}=\sqrt{2} M_{H} .
$$

The comparison between Equation (16) and Equation (11) shows that this result is obviously wrong. The question now is whether the assumption Equation (12) is wrong or our understanding of it is incomplete. If we accept Equation (12), the only thing we can do is to reinterpret the flavor eigenstates of the charged leptons.

The form of the Lagrangian density given by Equation (4) indicates that the basic spinor fermion fields are not 4-component Dirac spinors, but rather their left and right-handed projections. Thus, for the weak interactions, the elementary entities are states of chirality with zero mass [9]. Based on these viewpoints, we propose a hypothesis here that the flavor eigenstates of the charged leptons are the superposition states of left-handed and right-handed Weyl spinors before electroweak symmetry breaking, i.e.,

$$
\psi_{\ell}=\sqrt{2}\left(\cos \theta_{\ell} \ell_{L}+\sin \theta_{\ell} \ell_{R}\right) .
$$

where $\theta_{\ell}$ are three left-right mixing angles (Hereafter, we will limit the angles $\theta_{\ell}$ to $0 \leq \theta_{\ell} \leq \pi / 2$.), and factor $\sqrt{2}$ is to ensure that Equation (17) is the same as Equation (3) when $\cos \theta_{\ell}=\sin \theta_{\ell}=1 / \sqrt{2} . \ell_{L}$ and $\ell_{R}$ refer to the 
elementary entities, the left- and right-handed Weyl spinors, respectively.

By using Equation (17), the left- and right-handed charged leptons given in Equation (1) and Equation (2) should be replaced by

$$
\begin{aligned}
& L_{\ell}^{\prime}=\frac{1}{2}\left(1+\gamma_{5}\right)\left(\begin{array}{l}
v_{\ell} \\
\psi_{\ell}
\end{array}\right)=\left(\begin{array}{l}
v_{\ell} \\
\psi_{\ell}
\end{array}\right)_{L}, \\
& R_{\ell}^{\prime}=\frac{1}{2}\left(1-\gamma_{5}\right) \psi_{\ell}=\left(\psi_{\ell}\right)_{R} .
\end{aligned}
$$

Consequently, the Yukawa coupling in Equation (4) becomes

$$
\mathscr{L}_{Y}=-\frac{g}{\sqrt{2}} \frac{M_{H}}{M_{W}}\left(\overline{L_{\ell}^{\prime}} \phi R_{\ell}^{\prime}+\overline{R_{\ell}^{\prime}} \phi^{\dagger} L_{\ell}^{\prime}\right) \text {. }
$$

By using Equation (18) and Equation (19), the Lagrangian density which characterizes the coupling of leptons to gauge fields and Higgs field in Equation (4) changes to be

$$
\begin{aligned}
\mathscr{L}_{\ell}^{\prime}= & +i \frac{g}{\sqrt{2}}\left[W_{\mu}^{+} \overline{\left(v_{\ell}\right)_{L}} \gamma_{\mu}\left(\psi_{\ell}\right)_{L}+W_{\mu}^{-} \overline{\left(\psi_{\ell}\right)_{L}} \gamma_{\mu}\left(v_{\ell}\right)_{L}\right] \\
& -\frac{i}{2} \sqrt{g^{2}+g^{\prime 2}} Z_{\mu}\left[\overline{\left(v_{\ell}\right)_{L}} \gamma_{\mu}\left(v_{\ell}\right)_{L}-\overline{\left(\psi_{\ell}\right)_{L}} \gamma_{\mu}\left(\psi_{\ell}\right)_{L}+2 \sin ^{2} \theta_{W} \bar{\psi}_{\ell} \gamma_{\mu} \psi_{\ell}\right] \\
& -i \frac{g g^{\prime}}{\sqrt{g^{2}+g^{\prime 2}}} A_{\mu} \bar{\psi}_{\ell} \gamma_{\mu} \psi_{\ell}-\frac{g}{\sqrt{2}} \frac{M_{H}}{M_{W}}\left(\overline{L_{\ell}^{\prime}} \phi R_{\ell}^{\prime}+\overline{R_{\ell}^{\prime}} \phi^{\dagger} L_{\ell}^{\prime}\right) .
\end{aligned}
$$

In Equation (20), all the terms except the Yukawa term do not involve cross term of left- and right-handed states. Thus, in these terms, according to quantum principles, it can be seen from Equation (17) that the left-handed fermion state $\left(\psi_{\ell}\right)_{L}=\sqrt{2} \cos \theta_{\ell} \ell_{L}$ and $\ell_{L}$ correspond to the same state, and for the same reason, the right-handed fermion state $\left(\psi_{\ell}\right)_{R}=\sqrt{2} \sin \theta_{\ell} \ell_{R}$ and $\ell_{R}$ correspond to the same state, too. However, in the Yukawa term, because of the interference between left-handed state $\left(\psi_{\ell}\right)_{L}$ and right-handed state $\left(\psi_{\ell}\right)_{R}$, the coefficients $\sqrt{2} \cos \theta_{\ell}$ and $\sqrt{2} \sin \theta_{\ell}$ are indispensable.

Based on the above considerations, after parameterization and unitary gauge transformation, replacing $\phi$ by

$$
\left(\begin{array}{c}
0 \\
v+\eta
\end{array}\right)
$$

where $\eta$ is the real Higgs field. The Yukawa term in Equation (20) changes to be

$$
\mathscr{L}_{Y}=-\frac{g}{\sqrt{2}} \frac{M_{H}}{M_{W}} \sin \left(2 \theta_{\ell}\right)(v+\eta) \bar{\ell} \ell .
$$

Meanwhile, Equation (20) becomes

$$
\begin{aligned}
\mathscr{L}_{\ell}^{\prime}= & +i \frac{g}{\sqrt{2}}\left[W_{\mu}^{+} \overline{\left(v_{\ell}\right)_{L}} \gamma_{\mu} \ell_{L}+W_{\mu}^{-} \bar{\ell}_{L} \gamma_{\mu}\left(v_{\ell}\right)_{L}\right] \\
& -\frac{i}{2} \sqrt{g^{2}+g^{\prime 2}} Z_{\mu}\left[\overline{\left(v_{\ell}\right)_{L}} \gamma_{\mu}\left(v_{\ell}\right)_{L}-\bar{\ell}_{L} \gamma_{\mu} \ell_{L}+2 \sin ^{2} \theta_{W} \bar{\ell} \gamma_{\mu} \ell\right] \\
& -i \frac{g g^{\prime}}{\sqrt{g^{2}+g^{\prime 2}}} A_{\mu} \bar{\ell} \gamma_{\mu} \ell-\frac{g}{\sqrt{2}} \frac{M_{H}}{M_{W}} \sin \left(2 \theta_{\ell}\right)(v+\eta) \bar{\ell} \ell .
\end{aligned}
$$


This result is completely consistent with the standard WS model.

From Equation (22), we can obtain the masses of the charged leptons as

$$
m_{\ell}=\sqrt{2} M_{H} \sin \left(2 \theta_{\ell}\right) .
$$

Equation (24) shows that the difference of masses among the charged leptons of three generations comes from the different left-right mixing angles $\theta_{\ell}$ of their flavor eigenstates.

Comparing Equation (11) with Equation (24), the usual Yukawa coupling constant $G_{\ell}$ is given by

$$
G_{\ell}=\frac{\sqrt{2}}{v} M_{H} \sin \left(2 \theta_{\ell}\right)=\frac{g}{\sqrt{2}} \frac{M_{H}}{M_{W}} \sin \left(2 \theta_{\ell}\right) .
$$

From Equation (24), we can obtain

$$
\sin \left(2 \theta_{\ell}\right)=\frac{m_{\ell}}{\sqrt{2} M_{H}},
$$

where $m_{\ell} \ll \sqrt{2} M_{H}$ means $\theta_{\ell} \sim m_{\ell} / 2 \sqrt{2} M_{H} \ll 1$. Now put $m_{e} \sim 0.511 \mathrm{MeV}$, $m_{\mu} \sim 105.66 \mathrm{MeV}, m_{\tau} \sim 1776.86 \mathrm{MeV}$ and $M_{H} \sim 125 \mathrm{GeV}$, one can obtain the magnitude of the three left-right mixing angles are about

$$
\begin{aligned}
\theta_{e} & \sim 1.45 \times 10^{-6}, \\
\theta_{\mu} & \sim 2.99 \times 10^{-4}, \\
\theta_{\tau} & \sim 5.03 \times 10^{-3} .
\end{aligned}
$$

This result implies that the charged leptons, as the superposition states of leftand right-handed elementary Weyl spinors, have an extreme left-right asymmetry. It is noteworthy here that the existence of left-right asymmetry should be related to the destruction of parity conservation in weak interactions [10].

\section{Conclusion}

The present paper mainly discusses the origin of the masses of charged leptons. The proposed model is based on two basic hypotheses: the first one is that there exists a universal constant of the Yukawa coupling term, this constant has the same order of magnitude as the gauge coupling constant; the other one is that the flavor eigenstates of the charged leptons are the superposition states of left-handed and right-handed Weyl spinors with different weights before electroweak symmetry breaking. Based on these two hypotheses, it is found that the difference in masses among the three generations of charged leptons is due to the difference in the left-right mixing angles in the definition of the flavor eigenstates of the different charged leptons. In fact, the lines of thought presented in this paper may be applied to three generations of neutrinos and even to the three generations of quarks. The origin of neutrino masses is one of the most compelling evidences for physics beyond the Standard Model (SM) [11], and there is no reliable theory to explain it. We will discuss the problem of neutrinos in our forthcoming works. 


\section{Acknowledgements}

We thank the Editor and the referee for their comments.

\section{Conflicts of Interest}

The authors declare no conflicts of interest regarding the publication of this paper.

\section{References}

[1] Chatrchyan, S., et al. (2012) Physics Letters B, 716, 30-61.

[2] Aad, G., et al. (2012) Physics Letters B, 716, 1-29.

[3] Weinberg, S. (1967) Physical Review Letters, 19, 1264-1266. https://doi.org/10.1103/PhysRevLett.19.1264

[4] Salam, A. (1968) Elementary Particle Theory. In: Svartholm, N., Ed., Almquist and Forlag, Stockholm.

[5] Saldana-Salazar, U.J. (2016) Physical Review D, 93, 013002. https://doi.org/10.1103/PhysRevD.93.013002

[6] Abdalgabar, A. (2017) arXiv:1706.02313[hep-ph]

[7] King, S.F. (2018) Journal of High Energy Physics, 9, 069.

[8] de Varzielas, I.M. and King, S.F. (2019) Physical Review D, 99, 095029.

[9] Huang, K.S. (2009) Quarks, Leptons \& Gauge Fields. 2nd Edition, World Scientific Publishing Beijing Co Pte Ltd., Beijing.

[10] Lee, T.D. and Yang, C.N. (1956) Physical Review, 104, 254-258. https://doi.org/10.1103/PhysRev.104.254

[11] Tanabashi, M., et al. (2018) Physical Review D, 98, 030001. 